PROCEEDINGS OF THE

AMERICAN MATHEMATICAL SOCIETY

Volume 139, Number 7, July 2011, Pages 2615-2630

S 0002-9939(2010)10665-2

Article electronically published on December 8, 2010

\title{
TRANSVERSELY CANTOR LAMINATIONS AS INVERSE LIMITS
}

\author{
FERNANDO ALCALDE CUESTA, ÁLVARO LOZANO ROJO, \\ AND MARTA MACHO STADLER
}

(Communicated by Alexander N. Dranishnikov)

\begin{abstract}
We demonstrate that any minimal transversely Cantor compact lamination of dimension $p$ and class $C^{1}$ without holonomy is an inverse limit of compact branched manifolds of dimension $p$. To prove this result, we extend the triangulation theorem for $C^{1}$ manifolds to transversely Cantor $C^{1}$ laminations. In fact, we give a simple proof of this classical theorem based on the existence of $C^{1}$-compatible differentiable structures of class $C^{\infty}$.
\end{abstract}

\section{INTRODUCTION}

The covering map $f(z)=z^{2}$ defines a projective system $\mathbb{S}^{1} \stackrel{f}{\longleftarrow} \mathbb{S}^{1} \stackrel{f}{\longleftarrow} \ldots$, and its inverse limit $X=\lim _{\longleftarrow}\left(\mathbb{S}^{1}, f\right)=\left\{\left(z_{k}\right) \in \prod_{k \geq 0} \mathbb{S}^{1} \mid z_{k}^{2}=z_{k-1}\right\}$ is a Cantor fibre bundle over $\mathbb{S}^{1}$ endowed with a minimal free action of $\mathbb{R}$. This construction can be generalized by replacing the previous projective system with a sequence of compact $p$-manifolds and submersions between them.

Inverse limits have been used by R. F. Williams to model expanding attractors of diffeomorphisms [26]. In this case, the foliated space $X=\lim _{f}(M, f)$ is determined by a branched manifold $M$ and an expanding immersion $\leftarrow$. In another context, A. M. Vershik has shown that any Cantor minimal $\mathbb{Z}$-system is an inverse limit of directed graphs [24]. Similarly, J. Bellisard, R. Benedetti and J.-M. Gambaudo have proved that the continuous hull of any aperiodic and repetitive planar tiling is an inverse limit of branched flat surfaces [3. The proof is based on an inflation or zooming process, which replaces each tile with a pattern containing it. Using similar techniques, R. Benedetti and J.-M. Gambaudo have extended this result to $\mathbb{G}$-solenoids defined by free actions of a Lie group $\mathbb{G} 4$. Our aim is to generalize it for transversely Cantor minimal laminations without holonomy. Our result applies to examples by E. Ghys [10], E. Blanc [6] and the second author [16] which are not $\mathbb{G}$-solenoids.

Received by the editors February 25, 2010 and, in revised form, June 25, 2010.

2010 Mathematics Subject Classification. Primary 57R05, 57R30.

Key words and phrases. Lamination, triangulation, branched manifold, inverse limit.

This work was partially supported by MEC MTM2007-66262, UPV 00127.310-E-14790, EHU 06/05 and Xunta de Galicia INCITE07PXIE1R207053ES.

(C)2010 American Mathematical Society Reverts to public domain 28 years from publication 
Theorem 1.1 (inverse limit structure). Any minimal transversely Cantor compact lamination $(M, \mathcal{F})$ of dimension $p$ and class $C^{1}$ having trivial holonomy is an inverse limit of compact branched manifolds of dimension $p$.

In order to prove this theorem, we need to extend the classical triangulation theorem for $C^{1}$ manifolds 7, 8, 18, 27, 28, to transversely Cantor $C^{1}$ laminations:

Theorem 1.2 (existence of simplicial decompositions). Any transversely Cantor compact $C^{1}$ lamination $(M, \mathcal{F})$ admits a simplicial box decomposition $\mathcal{B}$.

In fact, we will give a simple proof of the existence of triangulations for $C^{1}$ manifolds, based on the existence of $C^{1}$-compatible differentiable structures of class $C^{\infty}$ given in 14; see Theorem 2.9, page 51 .

If $\mathcal{F}$ is minimal without holonomy, we can adapt the inflation process to our setting. This provides a sequence of box decompositions $\mathcal{B}^{(n)}$ such that $\mathcal{B}^{(1)}=$ $\mathcal{B}$ and $\mathcal{B}^{(n)}$ is obtained by inflation from $\mathcal{B}^{(n-1)}$. Thus we obtain a sequence of branched $p$-manifold $S_{n}$ collapsing each box of $\mathcal{B}^{(n)}$ to a plaque. Using inflation, we can also define cellular immersions $f_{n}: S_{n} \rightarrow S_{n-1}$. Now it is easy to see that $M=\lim _{(}\left(S_{n}, f_{n}\right)$.

As for Thurston's train tracks [23, any transverse invariant measure for $\mathcal{F}$ defines a simplicial cycle in $S_{n}$ such that the coefficient of each $p$-simplex is the measure of the transversal in the corresponding flow box. By the exhaustiveness of the inflation process, we shall prove the following result, which generalizes Theorem 5.1 of [4]:

Theorem 1.3 (transverse invariant measures). Let $(M, \mathcal{F})$ be an orientable transversely Cantor compact lamination of dimension $p$ and class $C^{1}$, which is obtained as an inverse limit of branched manifolds $S_{n}$ and cellular immersions $f_{n}: S_{n} \rightarrow S_{n-1}$. Then the set of transverse invariant measures for $\mathcal{F}$ is isomorphic to the inverse limit $\lim _{(}\left(H_{p}^{+}\left(S_{n} ; \mathbb{R}\right),\left(f_{n}\right)_{*}\right)$ where $H_{p}^{+}\left(S_{n} ; \mathbb{R}\right)$ is the positive cone of the $p$-th simplicial homology group $H_{p}\left(S_{n} ; \mathbb{R}\right)$ and $\left(f_{n}\right)_{*}: H_{p}\left(S_{n} ; \mathbb{R}\right) \rightarrow H_{p}\left(S_{n-1} ; \mathbb{R}\right)$ is the induced homomorphism.

\section{TRIANGUlations AND SIMPlicial DECOMPOSITIONS}

A compact space $M$ admits a $p$-dimensional lamination $\mathcal{F}$ of class $C^{r}, 1 \leq r \leq \infty$, if there exists a cover of $M$ by open sets $U_{i}$ (called flow boxes) homeomorphic to a product of an open ball $P_{i}$ in $\mathbb{R}^{p}$ centered at the origin 0 and a locally compact, separable and metrizable space $X_{i}$. In addition, if we denote by $\varphi_{i}: U_{i} \rightarrow P_{i} \times X_{i}$ the foliated charts, the changes of coordinates are given by

$$
\varphi_{i} \circ \varphi_{j}^{-1}(x, y)=\left(\varphi_{i j}^{y}(x), \sigma_{i j}(y)\right),
$$

where $\sigma_{i j}$ is a homeomorphism and $\varphi_{i j}^{y}$ is a $C^{r}$ diffeomorphism depending continuously on $y$ in the $C^{r}$-topology. The level sets $\varphi_{i}^{-1}\left(P_{i} \times\{y\}\right)$ and $\varphi_{i}^{-1}\left(\{x\} \times X_{i}\right)$ are the plaques and local transversals of $\mathcal{A}$ respectively. The lamination $\mathcal{F}$ is said to be transversely 0 -dimensional if $X=\coprod X_{i}$ is a 0 -dimensional space. We will always assume that $\mathcal{A}=\left\{\left(U_{i}, \varphi_{i}\right)\right\}_{i=1}^{m}$ is a good foliated atlas; i.e. $\mathcal{A}$ satisfies the following conditions:

(G1) the cover $\left\{U_{i}\right\}$ is finite;

(G2) the flow boxes $U_{i}$ are relatively compact;

(G3) if $U_{i} \cap U_{j} \neq \emptyset$, there is a flow box $U_{i j}$ containing $\overline{U_{i} \cap U_{j}}$, and therefore each plaque of $U_{i}$ intersects at most one plaque of $U_{j}$. 
Any cover by flow boxes $U_{i}$ admits a refinement by compact flow boxes $B_{\alpha}$, where $B_{\alpha}$ is a compact subset of $U_{i}$ such that $\varphi_{i}\left(U_{\alpha}\right)=D_{\alpha} \times C_{\alpha}$, where $D_{\alpha}$ is a closed ball in $P_{i}$ and $C_{\alpha}$ is a compact subset of $X_{i}$.

The leaves of $\mathcal{F}$ are $p$-dimensional polyhedra if the maps $\varphi_{i j}^{y}$ in (2.1) are $P L$ homeomorphisms, i.e. homeomorphisms that become linear up to subdivision. If the $P L$ structure of the plaques $\varphi_{i}^{-1}\left(P_{i} \times\{y\}\right)$ varies continuously (i.e. if $D$ is the star of a vertex in $\varphi_{i}^{-1}\left(P_{i} \times\{y\}\right)$ and $C$ is a neighborhood of $y \in X_{i}$, there is an embedding of $D \times C$ into $U_{i}$ which restricts to a linear inclusion of each simplex in $D \times\{z\}$ into $\left.\varphi_{i}^{-1}\left(P_{i} \times\{z\}\right)\right)$ and the linear restrictions of the maps $\varphi_{i j}^{y}$ depend continuously on $y$, we will say that $\mathcal{F}$ is a $P L$ lamination.

Each flow box $U_{i}$ admits a tangentially $C^{\infty}$-smooth Riemannian metric $g_{i}=\varphi_{i}^{*} g_{0}$ induced from a $C^{\infty}$-smooth Riemannian metric $g_{0}$ on $\mathbb{R}^{p}$. If $\mathcal{F}$ is $C^{\infty}$, we can glue together these local Riemannian metrics $g_{i}$ to a global one $g$ using a tangentially $C^{\infty}$-smooth partition of unity [17; see Proposition 2.8, page 37. Condition (G2) implies that $g$ is complete along the leaves. Now we will also suppose that:

(G4) each plaque $\varphi_{i}^{-1}\left(P_{i} \times\{y\}\right)$ is geodesically convex;

(G5) for each $x \in M$, the union $\operatorname{Star}(x, \mathcal{A})$ of all the plaques containing $x$ (called the star of $x$ with respect to $\mathcal{A}$ ) is contained in a geodesically convex set.

In fact, any compact $C^{1}$ lamination admits a complete Riemannian metric along the leaves, but we need $C^{2}$ smoothness in order to guarantee the local existence and uniqueness of geodesics.

Definition 2.1. Let $\left(K, \mathcal{F}_{K}\right)$ be a compact $P L$ lamination and $(M, \mathcal{F})$ a compact $C^{r}$ lamination. A map (resp. homeomorphism) $f: K \rightarrow M$ is said to be foliated if $f$ sends leaves into leaves and piecewise $C^{r}$ if the restriction of $f$ to each tangent simplex is of class $C^{r}$ (resp. a $C^{r}$ immersion). A $C^{r}$ triangulation of $(M, \mathcal{F})$ is a compact $P L$ lamination $\left(K, \mathcal{F}_{K}\right)$ together with a piecewise $C^{r}$ foliated homeomorphism $f:\left(K, \mathcal{F}_{K}\right) \rightarrow(M, \mathcal{F})$.

In the 0-dimensional transverse case, a $P L$ structure of this kind on a compact $C^{r}$ lamination can be easily derived from the following notion. In fact, as a consequence of the proof of Theorem 1.2, we will see that these two notions are equivalent:

Definition 2.2. A family of compact flow boxes $\mathcal{B}=\left\{\varphi_{i}: B_{i} \rightarrow D_{i} \times C_{i}\right\}_{i=1}^{m}$ is said to be a box decomposition of $(M, \mathcal{F})$ if

(D1) the family $\mathcal{B}$ covers $M$;

(D2) each transversal $C_{i}$ is a clopen set of $X$;

(D3) if $i \neq j$, the intersection of $B_{i}$ and $B_{j}$ agrees with the intersection of the vertical boundaries $\partial_{v} B_{i}=\varphi_{i}\left(\partial D_{i} \times C_{i}\right)$ and $\partial_{v} B_{j}=\varphi_{j}\left(\partial D_{j} \times C_{j}\right)$;

(D4) each plaque of $B_{i}$ meets at most one plaque of $B_{j}$;

(D5) the changes of coordinates are given by

$$
\varphi_{i} \circ \varphi_{j}^{-1}(x, y)=\left(\varphi_{i j}(x), \sigma_{i j}(y)\right) .
$$

If the plaques of $\mathcal{B}$ are $p$-simplices, each pair of plaques meets in a common face. In this case, we may suppose that the maps $\varphi_{i j}$ are linear and we will say that $\mathcal{B}$ is a simplicial box decomposition (or simply simplicial decomposition) of $(M, \mathcal{F})$.

As we have already said, our first aim is to show that the existence of triangulations for $C^{1}$ manifolds is still valid for transversely 0-dimensional $C^{1}$ laminations. If we replace the topology of $M$ with the (finer) leaf topology, then we obtain a 
$p$-manifold $\mathcal{M}$ of class $C^{1}$ whose connected components are the leaves of $\mathcal{F}$. But according to Theorem 2.10 on page 52 of [14, $\mathcal{M}$ is $C^{1}$ diffeomorphic to a $C^{\infty}$ manifold, and then one has local existence and uniqueness of geodesics in $\mathcal{M}$ (endowed with a suitable Riemannian metric $g$ ). Now we will triangulate $\mathcal{M}$ in the following way (using an approach inaugurated by A. Weil [26] which is only partially similar to that used in the classical proofs of $[18$ and $[27])$ :

1. Construction of the nerve of $\mathcal{A}$. Let $\mathcal{V}=\left\{P_{\alpha}\right\}_{\alpha \in A}$ be the open cover of $\mathcal{M}$ by plaques of $\mathcal{A}$. Its nerve $\mathcal{N}(\mathcal{V})$ is a simplicial complex containing all the combinatorial information about $\mathcal{V}$. Recall that a simplex $\left[\alpha_{0}, \alpha_{1}, \ldots, \alpha_{n}\right] \in \mathcal{N}(\mathcal{V})$ if and only if $P_{\alpha_{1}} \cap \cdots \cap P_{\alpha_{n}} \neq \emptyset$. Moreover, if we fix a well-ordering $\leq$ on $\mathcal{A}$, we may suppose that $\alpha_{0}<\alpha_{1}<\cdots<\alpha_{n}$.

2. Construction of a map $\Pi: \mathcal{N}(\mathcal{V}) \rightarrow \mathcal{M}$. For each plaque $P_{\alpha}$ in $U_{i}$, we associate its center $x_{\alpha}=\varphi_{i}(0, y)$ with the 0 -simplex $[\alpha] \in \mathcal{N}(\mathcal{V})$. For each 1simplex $\left[\alpha_{0}, \alpha_{1}\right] \in \mathcal{N}(\mathcal{V})$, the idea is to take the geodesic path $\gamma:[0,1] \rightarrow \mathcal{M}$ joining $\Pi\left(\left[\alpha_{0}\right]\right)=x_{\alpha_{0}}$ and $\Pi\left(\left[\alpha_{1}\right]\right)=x_{\alpha_{1}}$ and to define $\Pi(x)=\gamma\left(1-t_{0}\right)$ for each point $x=\left(t_{0}, t_{1}\right) \in\left[\alpha_{0}, \alpha_{1}\right]$. Reasoning inductively, the construction above can be extended to simplices of any dimension, and we will obtain a continuous surjection $\Pi: \mathcal{N}(\mathcal{V}) \rightarrow \mathcal{M}$

3. Triangulation of $\mathcal{M}$. If we choose the centers carefully, then $\Pi: \mathcal{N}(\mathcal{V}) \rightarrow \mathcal{M}$ becomes injective when restricted to $p$-simplices. Its images cover $\mathcal{M}$, but they do not form a triangulation because they can meet along $p$-dimensional convex polytopes. However, we have a decomposition of (each connected component of) $\mathcal{M}$ in $p$-dimensional convex polytopes. Now, to obtain a triangulation $\mathcal{K}$ of $\mathcal{M}$, it suffices to decompose each polytope into simplices by joining its center of mass with the faces of codimension one.

In the Borel case [5], the triangulation $\mathcal{K}$ of $\mathcal{M}$ induces a Borel family of triangulations $\mathcal{K}_{L}$ of the leaves $L \in \mathcal{F}$ (i.e. the centers of mass of $p$-simplices form a Borel transversal), and it is straightforward to construct a simplicial decomposition of $(M, \mathcal{F})$. In our case, we will directly construct a simplicial decomposition of $(M, \mathcal{F})$ in such a way that $\mathcal{K}_{L}$ varies continuously.

2.1. A compatible foliated structure of class $C^{\infty}$. Let us start by proving that any $C^{1}$ lamination admits a $C^{1}$-compatible differential structure of class $C^{\infty}$. To do so, we will adapt an analogous result for manifolds found in [14] (see Theorem 2.9, page 51) using the same technique as in Chapter II of [17]:

Lemma 2.3. Let $(M, \mathcal{F})$ be a $C^{1}$ lamination equipped with a $C^{1}$ foliated atlas $\mathcal{A}$. Then there exists a $C^{\infty}$ foliated atlas $C^{1}$-equivalent to $\mathcal{A}$ (i.e. the identity map is a foliated $C^{1}$ diffeomorphism).

Proof. By Zorn's lemma, there is a maximal open subset $V \subset M$ such that $\mathcal{F}$ induces a $C^{\infty}$ lamination $\left.\mathcal{F}\right|_{V}$ on $V$ up to a foliated $C^{1}$ diffeomorphism. In other words, the foliated charts of the $C^{\infty}$ lamination $\left.\mathcal{F}\right|_{V}$ are foliated charts of the $C^{1}$ lamination $\mathcal{F}$. We must prove $V=M$. If $V \neq M$, there is a foliated chart $\varphi: U \rightarrow P \times X$ of $(M, \mathcal{F})$ such that $U \cap V \neq \emptyset$ and $U \not \subset V$. Let $W=U \cap V$ and $W^{\prime}=\varphi(W)$. There are now two $C^{\infty}$-foliated structures on $W$ : one induced from $V$ and the other induced by $\varphi$. We shall replace the foliated chart $\varphi: U \rightarrow P \times X$ with another foliated chart $\psi: U \rightarrow P \times X$ such that $\left.\psi\right|_{W}: W \rightarrow W^{\prime}$ is a foliated $C^{\infty}$ diffeomorphism. In this case, the foliated chart $\psi: U \rightarrow P \times X$ could be added to the foliated atlas of $V$, and $V$ would not be maximal. 
To construct $\psi$, we start by showing how to obtain a neighborhood $\mathcal{N}$ of $\left.\psi\right|_{W}$ in $C^{1}\left(W, W^{\prime}\right)$. By paracompactness, $W$ has a locally finite cover $\left\{K_{i}\right\}$ by compact flow boxes. The compactness of $K_{i}$ implies that there is $\varepsilon_{i}>0$ such that $d(y, U-W)>\varepsilon_{i}$ for each $y \in K_{i}$ (where $d$ is a distance function on $U$ ). Let $\mathcal{N}$ be the set of maps $h \in C^{1}\left(W, W^{\prime}\right)$ such that

$$
\left\|h(y)-\left.\varphi\right|_{W}(y)\right\|<\varepsilon_{i} \quad \text { and } \quad\left\|d h(y)-d\left(\left.\varphi\right|_{W}\right)(y)\right\|<\varepsilon_{i}
$$

for each $y \in K_{i}$. Then $h$ is near $\left.\varphi\right|_{W}$ on each compactum. Moreover, each map $h \in \mathcal{N}$ can be extended to a map $T(h): U \rightarrow P \times X$ defined by

$$
T(h)= \begin{cases}h & \text { over } W, \\ \varphi & \text { over } U-W,\end{cases}
$$

and the resulting map $T: \mathcal{N} \rightarrow C^{1}(U, P \times X)$ is continuous (see [14, Lemma 2.8, page 50). Since $T\left(\left.\varphi\right|_{W}\right)=\varphi$ is a foliated $C^{1}$ diffeomorphism, there is a neighborhood $\mathcal{N}_{0} \subset \mathcal{N}$ of $\left.\varphi\right|_{W}$ whose elements are $C^{1}$ diffeomorphisms from $U$ to $P \times X$. By Approximation Theorem 2.11 of [17, there is a foliated $C^{\infty}$ diffeomorphism $\psi_{0}: W \rightarrow W^{\prime}$ in $\mathcal{N}_{0}$, and the required foliated chart is given by $\psi=T\left(\psi_{0}\right)$.

Remark 2.4. As a consequence of the lemma above, any compact $C^{1}$ lamination $(M, \mathcal{F})$ admits complete Riemannian metrics $g$ such that the local existence and uniqueness theorem holds for geodesics on the leaves.

2.2. Existence of an adapted atlas. In order to ensure the transverse continuity of the triangulations of the leaves, we should prevent disjoint plaques from meeting when they move transversely. With this idea in mind, we shall refine the classic definition of a good atlas:

Lemma $2.5(20])$. Let $(M, \mathcal{F})$ be a compact $C^{1}$ lamination and $g$ a Riemannian metric along the leaves. Then there exists a good foliated atlas $\mathcal{A}$ such that:

(A1) For every $x \in M, \operatorname{Star}(x, \mathcal{A})$ is contained in a geodesically convex set.

(A2) Boundaries of plaques intersect transversely or not at all.

Proof. Let $\mathcal{A}=\left\{\left(U_{i}, \varphi_{i}\right)\right\}_{i=1}^{m}$ be a good foliated atlas. Suppose that the plaques are geodesic balls of sufficiently small radius $r>0$ such that $\mathcal{A}$ verifies condition (A1). To prove our claim, we will use the method described by A. Phillips and D. Sullivan in [20]. The key idea is to eliminate tangencies by replacing the plaques that are involved with larger ones (see Figure 1).

Without loss of generality, we may assume that $\mathcal{A}$ is a refinement of a foliated atlas with the same number of charts $\hat{\varphi}_{i}: \hat{U}_{i} \rightarrow \hat{P}_{i} \times \hat{X}_{i}$, where $\overline{U_{i}} \subset \hat{U}_{i},\left.\hat{\varphi}_{i}\right|_{U_{i}}=\varphi_{i}$, $\overline{X_{i}} \subset \hat{X}_{i}$ and $\hat{P}_{i}$ is a concentric ball to $P_{i}$ of radius $2 r$. Since $\mathcal{A}$ fulfills (A1), we must inductively construct new flow boxes $U_{i}$ satisfying (A2). Obviously the

a)

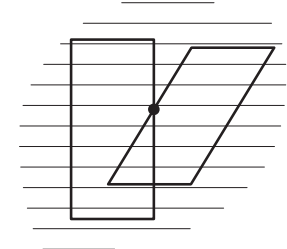

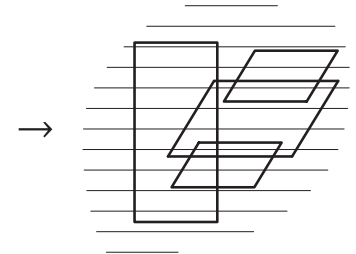

b)

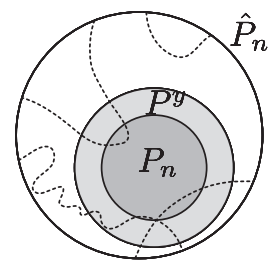

Figure 1. a) Removing tangencies, b) choice of $P^{y}$ 
partial cover $\mathcal{W}_{1}=\left\{U_{1}\right\}$ satisfies both conditions. Now let us suppose that $\bigcup_{i=1}^{n-1} U_{i}$ is covered by a family of flow boxes $\mathcal{W}_{n-1}=\left\{W_{1}, \ldots, W_{l_{n-1}}\right\}$ satisfying (A1) and (A2). For each point $y \in X_{n}$, there is a ball $P^{y}$ contained in the plaque of $\hat{U}_{n}$ passing through $y$ such that the boundary of $P^{y}$ meets transversely the boundary of all the plaques of $\mathcal{W}_{n-1}$. Indeed, if the boundary of the plaque of $U_{n}$ through $y$ has no tangencies, we define $P^{y}=\varphi_{k}^{-1}\left(P_{n} \times\{y\}\right)$. In the other case, the tangencies can be eliminated by expanding $P_{n} \times\{y\}$ into a ball of radius $r<\hat{r}<2 r$. Now we take $C_{y} \subset \overline{C_{y}} \subset \hat{X}_{n}$ a neighborhood of $y$ such that the boundary of $\hat{\varphi}_{n}^{-1}\left(P^{y} \times\{z\}\right)$ transversely meets the boundary of all plaques of $\mathcal{W}_{n-1}$ for every $z \in C_{y}$. As $X_{n}$ is relatively compact in $\hat{X}_{n}$, it is covered by a finite number of such open sets $C_{y_{1}}, \ldots, C_{y_{l}}$. Thus we obtain a finite family of flow boxes $W_{l_{n-1}+j}=\hat{\varphi}_{n}^{-1}\left(P^{y_{j}} \times C_{y_{j}}\right)$ with $j=1, \ldots, l$. The boundaries of the plaques of these flow boxes can still meet tangentially, but then $P^{y_{j}}=P^{y_{k}}$ and $C_{y_{j}} \cap C_{y_{k}} \neq \emptyset$ for $j \neq k$. To eliminate the tangencies, it is now enough to expand $P^{y_{j}}$ again slightly. Therefore, the family of flow boxes $\mathcal{W}_{n}=\mathcal{W}_{n-1} \cup\left\{W_{l_{n-1}+1}, W_{l_{n-1}+2}, \ldots, W_{l_{n-1}+l}\right\}$ covers $\bigcup_{i=0}^{n} U_{n}$ and the boundaries of its plaques have no tangencies. As the diameter of plaques is less than $4 r, \operatorname{Star}\left(x, \mathcal{W}_{n}\right)$ is contained in a geodesic ball of radius $8 r$. Finally, if we choose a sufficiently small $r>0$, we can ensure that the ball is geodesically convex. In a finite number of steps, we will conclude the proof.

Using smaller radii, we may replace condition (A1) with the stronger one (A1') described below. In fact, in the 0-dimensional case we may add some other useful conditions:

Lemma 2.6. Let $(M, \mathcal{F})$ be a transversely 0 -dimensional compact $C^{1}$ lamination. Then there exists a good foliated atlas $\mathcal{A}$ such that:

$\left(\mathrm{A} 1^{\prime}\right)$ Any star $\operatorname{Star}(\operatorname{Star}(x, \mathcal{A}), \mathcal{A})$ is contained in a geodesically convex set.

$\left(\mathrm{A} 2^{\prime}\right)$ Boundaries of plaques intersect transversely or not at all.

$\left(\mathrm{A}^{\prime}\right)$ Local transversals $X_{i}$ are compact.

(A4') Holonomy transformations $\sigma_{i j}$ are defined on clopen sets.

2.3. Foliated nerve of a foliated atlas. If $M$ is endowed with the leaf topology, then $\mathcal{A}$ defines a cover $\mathcal{V}$ of $\mathcal{M}$. Its nerve $\mathcal{N}(\mathcal{V})$ is the disjoint union of the nerves of the induced covers on the leaves. If $\mathcal{F}$ is transversely 0-dimensional, $\mathcal{N}(\mathcal{V})$ comes from a $P L$ foliated space by simplicial complexes that will be denoted by $\mathcal{N}_{\mathcal{F}}(\mathcal{A})$.

Definition 2.7. Let $\mathcal{A}=\left\{\left(U_{i}, \varphi_{i}\right)\right\}_{i=1}^{m}$ be a good foliated atlas of $(M, \mathcal{F})$ as in Lemma 2.6. We will call a foliated nerve of $\mathcal{A}$ the union $\mathcal{N}_{\mathcal{F}}(\mathcal{A})$ of the family $\mathcal{B}(\mathcal{A})=$ $\left\{\Delta \times X_{\Delta}\right\}$, where $\Delta=\left[i_{0}, i_{1}, \ldots, i_{k}\right] \in \mathcal{N}(\mathcal{U})$ corresponds to a finite sequence $i_{0}<$ $i_{1}<\cdots<i_{k}$ such that $\bigcap_{l=0}^{k} U_{i_{l}} \neq \emptyset$ and $X_{\Delta}=\bigcap_{l=0}^{k} \operatorname{dom} \sigma_{i_{0} i_{l}}$. The space $\mathcal{N}_{\mathcal{F}}(\mathcal{A})$ is endowed with the weak topology which makes each product $\Delta \times X_{\Delta}$ a flow box.

Lemma 2.8. The foliated nerve $\mathcal{N}_{\mathcal{F}}(\mathcal{A})$ is a compact $P L$ foliated space given by the decomposition $\mathcal{B}(\mathcal{A})$.

Proof. In order to prove that $\mathcal{N}_{\mathcal{F}}(\mathcal{A})$ is a $P L$ foliated space, or equivalently that $\mathcal{B}(\mathcal{A})$ is a simplicial box decomposition, we must show that the simplicial structure is transversely preserved around any vertex. We fix a vertex $x$ in the intersection of a transversal $[i] \times X_{i}$ (identified with $X_{i}$ ) and a box $\Delta \times X_{\Delta}$. If we write $X_{\Delta}^{x}=X_{i} \cap\left(\Delta \times X_{\Delta}\right)=\sigma_{i_{0} i}\left(X_{\Delta} \cap \operatorname{dom} \sigma_{i_{0} i}\right)$, then $\Delta \times\{y\} \in \operatorname{Star}(y, \mathcal{A})$ for all $y \in X_{\Delta}^{x}$. Therefore, $\operatorname{Star}(x, \mathcal{A}) \times\{y\} \subset \operatorname{Star}(y, \mathcal{A})$ for all $y \in \bigcap_{\Delta \in \operatorname{Star}(x, \mathcal{A})} X_{\Delta}^{x}$. 

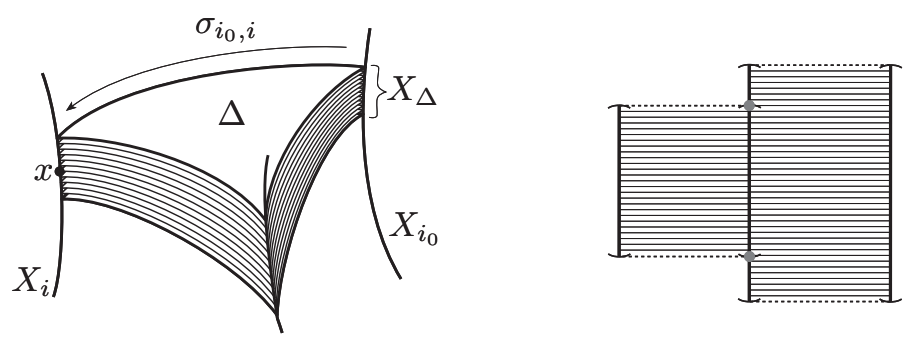

FiguRE 2. Local structure and emergence of new simplices

However, the equality may not hold true (see Figure 2). To solve this problem, it is enough to take the following clopen subset of $X_{i}$ :

$$
X^{x}=\bigcap_{\Delta \in \operatorname{Star}(x, \mathcal{A})} X_{\Delta}^{x}-\bigcup_{\Delta \in \mathcal{N}(\mathcal{U})-\operatorname{Star}(x, \mathcal{A})} X_{\Delta}^{x} .
$$

For each $y \in X^{x}$ we have

$$
\operatorname{Star}(y, \mathcal{A})=\operatorname{Star}(x, \mathcal{A}) \times\{y\}
$$

and thus the local simplicial structure does not change transversely.

If $\mathcal{B}^{n}(\mathcal{A})$ denotes the family of flow boxes $\Delta \times X_{\Delta}$ whose plaques have dimension $k \leq n$, then $\mathcal{N}_{\mathcal{F}}(\mathcal{A})$ is the union of the compact $P L$ foliated spaces $\mathcal{N}_{\mathcal{F}}^{n}(\mathcal{A})$ obtained from $\mathcal{B}^{n}(\mathcal{A})$. We will say that $\mathcal{N}_{\mathcal{F}}^{n}(\mathcal{A})$ is the $n$-skeleton of $\mathcal{N}_{\mathcal{F}}(\mathcal{A})$. Clearly, if we replace the weak topology on $\mathcal{N}_{\mathcal{F}}(\mathcal{A})$ by the (finer) leaf topology, we recover $\mathcal{N}(\mathcal{V})$.

2.4. From the foliated nerve to the lamination. Now it is easy to construct a continuous surjection $\Pi: \mathcal{N}(\mathcal{V}) \rightarrow \mathcal{M}$ using geodesic paths in $\mathcal{M}$ (which depends on the well-ordering fixed on $\mathcal{A}$ ). We will actually prove that $\Pi$ comes from a foliated map $\Pi_{\mathcal{F}}: \mathcal{N}_{\mathcal{F}}(\mathcal{A}) \rightarrow M$.

0-simplices: For each vertex $[i] \times\{y\} \in[i] \times X_{i}$, we define $\Pi_{\mathcal{F}}([i] \times\{y\})=x_{i}(y)$ as the center $x_{i}(y)=\varphi_{i}^{-1}(0, y)$ of the plaque $\varphi_{i}^{-1}\left(P_{i} \times\{y\}\right) \subset U_{i}$.

1-simplices: Fix a point $(x, y)$ in the simplex $[i, j] \times\{y\} \subset[i, j] \times \operatorname{dom} \sigma_{i j}$ with $i<j$. We know that $\operatorname{Star}\left(\operatorname{Star}\left(x_{i}(y), \mathcal{A}\right), \mathcal{A}\right)$ is contained in a geodesically convex set. Then there is a unique minimizing geodesic $\gamma_{i j}^{y}:[0,1] \rightarrow M$ joining $\Pi_{\mathcal{F}}([i] \times\{y\})=x_{i}(y)$ and $\Pi_{\mathcal{F}}\left([j] \times\left\{\sigma_{i j}(y)\right\}\right)=x_{j}\left(\sigma_{i j}(y)\right)$. We define

$$
\Pi_{\mathcal{F}}(x, y)=\gamma_{i j}^{y}\left(1-t_{0}(x)\right)=\gamma_{i j}^{y}\left(t_{1}(x)\right)
$$

where $t_{0}(x)$ and $t_{1}(x)$ are the barycentric coordinates of $x$. Obviously $\Pi_{\mathcal{F}}$ is a piecewise $C^{1}$ well-defined map over the 1 -skeleton $\mathcal{N}_{\mathcal{F}}^{1}(\mathcal{A})$ of $\mathcal{N}_{\mathcal{F}}(\mathcal{A})$.

$k$-simplices: If $\Pi_{\mathcal{F}}$ is defined over the $(k-1)$-skeleton of $\mathcal{N}_{\mathcal{F}}(\mathcal{A})$, we will consider a $k$-simplex $\Delta \times\{y\}=\left[i_{0}, i_{1}, \ldots, i_{k}\right] \times\{y\} \subset \Delta \times X_{\Delta}$ and a point $(x, y) \neq\left[i_{0}\right] \times\{y\}$. The half-line from $\left[i_{0}\right]$ to $x$ meets $\partial_{0} \Delta=\left[i_{1}, i_{2}, \ldots, i_{k}\right]$ in a unique point $x^{\prime}$ with barycentric coordinates $\left(0, t_{1} / 1-t_{0}, \ldots, t_{k} / 1-t_{0}\right), t_{i}$ being the $i$-th barycentric coordinate of $x$. Since $\left(x^{\prime}, \sigma_{i_{0} i_{1}}(y)\right)$ belongs to the $(k-1)$-simplex $\partial_{0} \Delta \times\left\{\sigma_{i_{0} i_{1}}(y)\right\} \subset$ $\partial_{0} \Delta \times \bigcap_{l=1}^{k} \operatorname{dom} \sigma_{i_{0} i_{l}}$, its image $\Pi_{\mathcal{F}}\left(x^{\prime}, \sigma_{i_{0} i_{1}}(y)\right)$ is well defined. As above, there is a unique minimizing geodesic $\gamma_{i_{0} \ldots i_{k}}^{y}:[0,1] \rightarrow M$ joining $\Pi_{\mathcal{F}}\left(\left[i_{0}\right] \times\{y\}\right)$ and $\Pi_{\mathcal{F}}\left(x^{\prime}, \sigma_{i_{0} i_{1}}(y)\right)$. Now we define

$$
\Pi_{\mathcal{F}}(x, y)=\gamma_{i_{0} \ldots i_{k}}^{y}\left(1-t_{0}(x)\right) .
$$



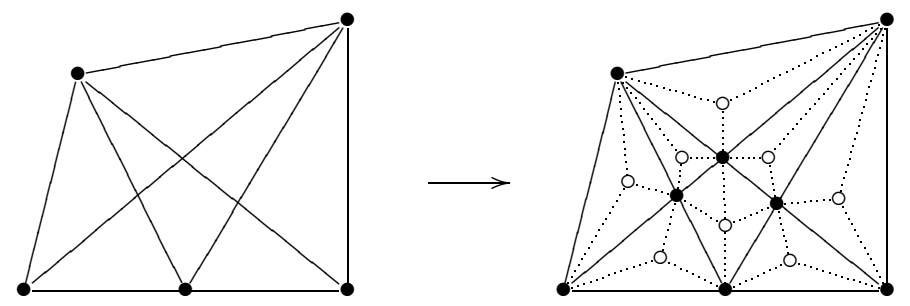

Figure 3. Polytope and simplicial decompositions

Clearly this map coincides with $\Pi_{\mathcal{F}}$ over each face $\partial_{0} \Delta \times\left\{\sigma_{i_{0} i_{1}}(y)\right\}$ and $\partial_{l} \Delta \times\{y\}=$ $\left[i_{0}, \ldots, \widehat{i_{l}}, \ldots, i_{k}\right] \times\{y\}$ of $\Delta \times\{y\}, 1 \leq l \leq k$. It provides a piecewise $C^{1}$ well-defined map over the $k$-skeleton $\mathcal{N}_{\mathcal{F}}^{k}(\mathcal{A})$ of $\mathcal{N}_{\mathcal{F}}(\mathcal{A})$.

By induction, we obtain the announced map $\Pi_{\mathcal{F}}: \mathcal{N}_{\mathcal{F}}(\mathcal{A}) \rightarrow M$. If we endow $\mathcal{N}_{\mathcal{F}}(\mathcal{A})$ and $M$ with the leaf topology, then $\Pi_{\mathcal{F}}$ agrees with $\Pi$. This implies that $\Pi_{\mathcal{F}}$ is a foliated surjection. In fact, $\Pi_{\mathcal{F}}$ is still surjective over the $p$-skeleton of $\mathcal{N}_{\mathcal{F}}(\mathcal{A})$, because the image of each $(p+1)$-simplex is the union of the images of its proper faces. We will resume this discussion in the following result:

Lemma 2.9. There is a piecewise $C^{1}$ foliated surjection $\Pi_{\mathcal{F}}: \mathcal{N}_{\mathcal{F}}^{p}(\mathcal{A}) \rightarrow M$.

2.5. Triangulation. The aim of this section is to complete the proof of Theorem 1.2. We first recall that, by carefully choosing the centers of the elements of $\mathcal{V}$, we can assure that the map $\Pi: \mathcal{N}(\mathcal{V}) \rightarrow \mathcal{M}$ is injective when restricted to each $p$-simplex. Unfortunately, we do not have a triangulation of $\mathcal{M}$ because the images of two $p$-simplices may intersect in a $p$-dimensional geodesically convex polytope. But, as we said before, we have a decomposition of $\mathcal{M}$ in geodesically convex polytopes. To obtain a triangulation of $\mathcal{M}$, it is enough to decompose each polytope into simplices by joining its center of mass with the $(p-2)$-dimensional faces (see Figure 3). Let us start by choosing good transversals made of centers:

Lemma 2.10. The map $\Pi_{\mathcal{F}}: \mathcal{N}_{\mathcal{F}}^{p}(\mathcal{A}) \rightarrow M$ is a piecewise $C^{1}$ injective immersion in restriction to each simplicial box $\Delta \times X_{\Delta}$.

Proof. It suffices to examine the case $\Delta=\left[i_{0}, i_{1}, \ldots, i_{p}\right]$. Notice that the map $\Pi_{\mathcal{F}}: \Delta \times X_{\Delta} \rightarrow M$ lifts to a linear map $\Upsilon: \Delta \times X_{\Delta} \rightarrow T(\mathcal{F})$ which makes the following diagram commutative:

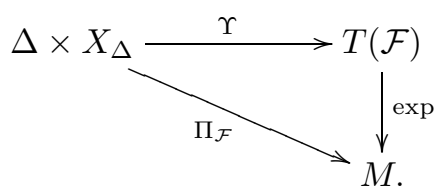

Indeed, since $\operatorname{Star}(x, \mathcal{A})$ is contained in a geodesic ball of sufficiently small radius, we can ensure that the exponential map exp $: T(\mathcal{F}) \rightarrow M$ has a local section. For any $y \in X_{\Delta}$, the set $\Upsilon(\Delta \times\{y\})$ is the convex hull of the images by $\Upsilon$ of the vertices of $\Delta \times\{y\}$ with $\Upsilon\left(\left[i_{0}\right] \times\{y\}\right)=0$. Let $B_{\Delta}$ denote the compact set of points $y \in X_{\Delta}$ such that $\Upsilon\left(\left[i_{1}\right] \times\{y\}\right), \ldots, \Upsilon\left(\left[i_{p}\right] \times\{y\}\right)$ are not linearly independent, or equivalently $\left.\Pi_{\mathcal{F}}\right|_{\Delta \times\{y\}}$ is not injective. Each point $y$ has a clopen $X^{y} \subset X_{\Delta}$ such that $\operatorname{Star}(z, \mathcal{A})=\operatorname{Star}(y, \mathcal{A})$ for all $z \in X^{y}$. Thus we can replace the centers 
$\Pi_{\mathcal{F}}([i] \times\{x\})=x_{i}(y)$ and the transversals $\Pi_{\mathcal{F}}\left([i] \times X_{\Delta}^{y}\right) \subset\left\{x_{i}(y)\right\} \times X_{i}$ with nearby points $\widehat{x}_{i}(y)$ and the transversals $\widehat{X}_{i} \subset P_{i} \times X_{i}$ (which are projectable over $X^{y}$ ) such that $\widehat{\Upsilon}\left(\left[i_{1}\right] \times\{z\}\right), \ldots, \widehat{\Upsilon}\left(\left[i_{k}\right] \times\{z\}\right)$ are linearly independent for all $z \in X^{y}$. By compacity of $B_{\Delta}$, we can reduce it to the empty set in a finite number of steps.

From the previous lemma, $M$ is covered by a finite family of simplicial boxes. As in the classic case, it is not a triangulation, but we have the following result:

Theorem 2.11. Any transversely 0 -dimensional compact $C^{1}$ lamination admits a decomposition by flow boxes with geodesically convex plaques.

Proof. Let $\Delta \times X_{\Delta}$ and $\Delta^{\prime} \times X_{\Delta^{\prime}}$ be two simplicial boxes whose images by $\Pi_{\mathcal{F}}$ have no empty intersection. By the lemma above, we can identify each of them with its image. In general, the intersection of (the images of) these boxes is not foliated as a product, but the (images of the) plaques intersect in geodesically convex sets. Again using the local invariance of the simplicial structure around any vertex (see Lemma 2.8), we can decompose it in a finite number of compact flow boxes homeomorphic to the product of a geodesically convex set and a clopen set. By construction, the changes of coordinates are linear maps (continuously depending on the transverse coordinate $y$ ) which are completely determined by the choice of the metric $g$. However, we can modify $g$ in order to reparametrize geodesics along the vertical faces of flow boxes in such a way that the changes of coordinates no longer depend on $y$.

Proof of Theorem 1.2. By the previous theorem, $M$ admits a box decomposition whose plaques are geodesically convex polytopes. Given one of these flow boxes, we can triangulate all the plaques at once, joining its centers of mass (which form a local transversal) with the codimension one faces (which cover the vertical boundary) by geodesics. In this way, we decompose the flow box in a finite union of simplicial flow boxes, as in Figure 4. To conclude, we can proceed as before, assuming that the faces of the plaques are linearly homeomorphic to the faces of $\Delta$.

Corollary 2.12. Any transversely 0 -dimensional compact $C^{1}$ lamination $(M, \mathcal{F})$ admits a $C^{1}$ triangulation.

Remark 2.13. There is another proof of Theorem 1.2 which is similar to Whitney's for manifolds (see [28, page 124). Here we will just offer an outline. First, recall that any compact $C^{1}$ lamination $(M, \mathcal{F})$ admits a leafwise $C^{1}$ topological embedding into a separable real Hilbert space (see [9], Theorem 11.4.4, page 299). In fact, if $\mathcal{F}$ has a total transversal of finite topological dimension $q$, then $M$ embeds into a Euclidean space $\mathbb{R}^{2 m+1}$ with $m=p+q$ (according to Theorem 50.5 of [19]).

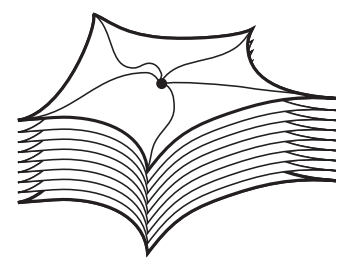

FIGURE 4. Breaking up into simplicial boxes 
Now consider the tangent bundle $T \mathcal{F}$ defined by putting the tangent spaces to the leaves at each point. Then $T \mathcal{F}$ extends to a $C^{0}$ plane field $\tau$ on $\mathbb{R}^{2 m+1}$. Given a triangulation of $\mathbb{R}^{2 m+1}$, by taking a sufficiently fine subdivision and applying Thurston's Jiggling Lemma 22, we may assume that $\tau$ (and therefore $\mathcal{F}$ ) is in general position in the sense of 22, page 219. We may also suppose that the angle at which each leaf intersects a simplex of dimension $\leq m+1$ is uniformly bounded from below. This makes it possible to apply the triangulation procedure of 28 ] and to obtain a transversely measurable family of triangulations on the leaves of $\mathcal{F}$. In fact, we are proposing to adapt the Heitsch-Lazarov construction [13] to transversely finite-dimensional laminations. In the 0 -dimensional case, arguing as before, it is possible to show that $M$ intersects no simplices of dimension $\leq p$ (compare to [27, page 125). Moreover, for each $(p+1)$-simplex which meets $M$, each leaf $L$ intersects this simplex at a unique point (with not too small an angle). This allows us to construct a PL submanifold of dimension $p$ which approximates $L$ closely enough to obtain a triangulation of $L$ by normal projection.

\section{INFLATION AND INVERSE LIMITS}

In this section, we will complete the proof of Theorem 1.1. Using the properties of $\mathcal{F}$, namely minimality and triviality of the holonomy, we will adapt the inflation or zooming process described in $[3$ and [4. Let us start by recalling these properties. First, $\mathcal{F}$ is minimal if all the leaves are dense. On the other hand, we can define the triviality of the holonomy making use of the local Reeb stability theorem. Thus, we say that $\mathcal{F}$ has trivial holonomy if for every point $x \in M$ and for every geodesic ball $D_{x}$ centered at $x$ in the leaf passing through $x$, there is a neighborhood $C_{x}$ of $x$ in a total transversal and a foliated embedding of $D_{x} \times C_{x}$ into $M$.

\subsection{Inflation.}

Definition 3.1. Let $\mathcal{B}$ and $\mathcal{B}^{\prime}$ be two box decompositions of $(M, \mathcal{F})$. We will say that $\mathcal{B}^{\prime}$ is inflated from $\mathcal{B}$ if

(I1) For each point $x$ in the intersection of two boxes $B \in \mathcal{B}$ and $B^{\prime} \in \mathcal{B}^{\prime}$, the transversal of $B^{\prime}$ passing through $x$ is contained in the transversal of $B$.

(I2) The vertical boundary of each box $B^{\prime} \in \mathcal{B}^{\prime}$ is contained in the vertical boundary of $\mathcal{B}$; that is, $\partial_{v} B^{\prime} \subset \bigcup_{B \in \mathcal{B}} \partial_{v} B$.

(I3) For each box $B^{\prime} \in \mathcal{B}^{\prime}$, there is a box $B \in \mathcal{B}$ such that $B \cap B^{\prime} \neq \emptyset$ and $\partial_{v} B^{\prime} \cap \partial_{v} B=\emptyset$.

Theorem $3.2([3,4])$. Let $(M, \mathcal{F})$ be a transversely 0-dimensional compact lamination which is minimal and has trivial holonomy. For each box decomposition $\mathcal{B}$, there is a box decomposition $\mathcal{B}^{\prime}$ inflated from $\mathcal{B}$.

Proof. Let $\mathcal{C}=\bigsqcup_{i=1}^{m} C_{i}$ be the axis of $\mathcal{B}=\left\{B_{i}\right\}_{i=1}^{m}$. For each leaf $L \in \mathcal{F}$, the trace $\mathcal{G}=L \cap \mathcal{C}$ of $L$ is the 0 -skeleton of the corresponding leaf in the foliated space $\mathcal{N}_{\mathcal{F}}(\mathcal{B})$. Then $\mathcal{G}$ has a natural distance $d$ defined by means of the length of edge paths in the 1-skeleton of $\mathcal{N}_{\mathcal{F}}(\mathcal{B})$. Indeed, for all $x, y \in \mathcal{G}, d(x, y)$ is the minimum number of plaques $D_{1}, \ldots, D_{k}$ of $\mathcal{B}$ such that $x \in D_{1}, y \in D_{k}$ and $D_{i} \cap D_{i+1} \neq \emptyset$ for $1 \leq i<k$. Now $\mathcal{G}$ is quasi-isometric to the leaf $L$. 
Since $\mathcal{F}$ is minimal, any clopen set $\widehat{C}$ of $C_{1}$ meets all the leaves of $\mathcal{F}$. Therefore $\widehat{\mathcal{G}}=L \cap \widehat{C}$ is also quasi-isometric to $L$. Thus $\mathcal{G}$ and $\widehat{\mathcal{G}}$ are quasi-isometric. More precisely, $\widehat{\mathcal{G}}$ is an $R$-dense subset of $\mathcal{G}$, i.e. $\exists R>0$ such that $d(x, \widehat{\mathcal{G}})<R$ for all $x \in \mathcal{G}$. Moreover, if we choose a sufficiently small $\widehat{C}$, we can suppose that $\widehat{\mathcal{G}}$ is $r$-separated, i.e. $d(x, y) \geq r>0$ for all $x \neq y$ in $\widehat{\mathcal{G}}$.

For each point $x \in \widehat{\mathcal{G}}$, let $V_{x}$ be the set of points $y \in \mathcal{G}$ such that $d(y, x)=d(y, \widehat{\mathcal{G}})$. As $V_{x} \neq\{x\}$ is finite, $\widehat{D}_{x}=\bigcup_{y \in V_{x}} D_{y}$ is a compact subset of the leaf $L_{x}$ through $x$, which is not reduced to the plaque $D_{x}$. Now, since $\mathcal{F}$ has no holonomy, each point $x \in \widehat{C}$ has a clopen neighborhood $\widehat{C}_{x}$ such that the inclusion of $\widehat{D}_{x}$ into $L_{x}$ extends to an embedding of $\widehat{D}_{x} \times \widehat{C}_{x}$ into $M$. Its image $\widehat{B}_{x}=\bigcup_{y \in \widehat{C}_{x}} \widehat{D}_{y}$ is a compact flow box. Thus $\widehat{C}$ is covered by a finite number of pairwise $\operatorname{disjoint}$ sets $\widehat{C}_{1}, \ldots, \widehat{C}_{k}$ and $M$ admits a finite decomposition $\widehat{\mathcal{B}}=\left\{\widehat{B}_{1}, \ldots, \widehat{B}_{k}\right\}$ into compact flow boxes. It is obvious that $\widehat{\mathcal{B}}$ fulfills all the requirements of Definition 2.2 , except that the interior of two boxes $\widehat{B}_{i}$ and $\widehat{B}_{j}$ may intersect. In this case, there would be a holonomy transformation $\sigma_{i j}$ between the $\widehat{C}_{i}$ and $\widehat{C}_{j}$ and a decomposition of $\widehat{C}_{i}$ in clopen sets $\widehat{C}_{i}^{1}$ and $\widehat{C}_{i}^{2}$ such that:

i) the plaques $\widehat{D}_{i}$ through the points of $\widehat{C}_{i}^{1}$ do not meet $\widehat{B}_{j}$;

ii) the plaques $\widehat{D}_{i}$ and $\widehat{D}_{j}$ through the points $y_{i} \in \widehat{C}_{i}^{2}$ and $y_{j}=\sigma_{i j}\left(y_{i}\right) \in \widehat{C}_{j}$ meet along plaques $D_{x}$ associated to points $x \in V_{y_{i}} \cap V_{y_{j}}$ equidistant from $y_{i}$ and $y_{j}$.

Now we replace $\widehat{B}_{i}$ with $\widehat{B}_{i}^{1}$ and $\widehat{B}_{i}^{2}$, where $\widehat{B}_{i}^{1}$ is the union of all the plaques $\widehat{P}_{i}$ through the points of $\widehat{C}_{i}^{1}$ and where $\widehat{B}_{i}^{2}$ is obtained by removing the interiors of the plaques $D_{x}$ of the points $x \in V_{y_{i}} \cap V_{y_{j}}$ (see Figure 5). In a finite number of steps, we will obtain a new box decomposition $\mathcal{B}^{\prime}$ inflated from $\mathcal{B}$.

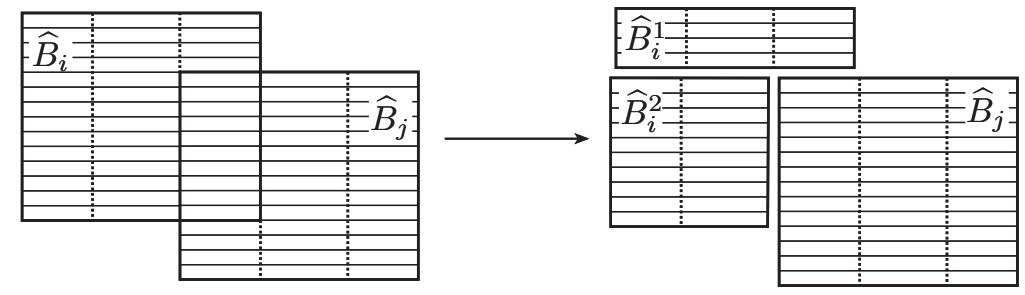

FIGURE 5. Cutting the intersections

Corollary 3.3. In the conditions of Theorem 3.2, for each box decomposition $\mathcal{B}$, there is a sequence of box decompositions $\mathcal{B}^{(n)}$ such that $\mathcal{B}^{(1)}=\mathcal{B}, \mathcal{B}^{(n+1)}$ is inflated from $\mathcal{B}^{(n)}$ and the intersection of the axes $\mathcal{C}^{(n)}$ is a point.

3.2. Branched manifolds. Here we will recall the definition of a branched manifold introduced by R. F. Williams [26]. Roughly speaking, they are CW-complexes with tangent space at all points as shown in Figure 6.

Definition 3.4 ([26]). A branched manifold of dimension $p$ and class $C^{r}$ is a locally compact metrizable and separable space $S$ endowed with:

(B1) a family of closed subsets $U_{i}$ of $S$ such that $S=\bigcup_{i} \stackrel{\circ}{U}_{i}$; 

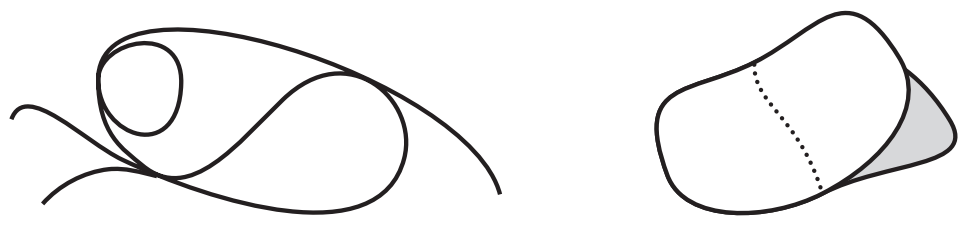

Figure 6. Branched manifolds

(B2) a finite family of closed subsets $D_{i j}$ of $U_{i}$ such that $\bigcup D_{i j}=U_{i}$;

(B3) a family of maps $\psi_{i}: U_{i} \rightarrow \mathbb{D}^{p}$ such that the maps $\left.\psi_{i}\right|_{D_{i j}}: D_{i j} \rightarrow \mathbb{D}^{p}$ are homeomorphisms and the changes of charts $\psi_{i} \circ \psi_{j}^{-1}$ are $C^{r}$-diffeomorphisms.

The set of points without any neighborhood homeomorphic to a disk is the singular part of $S$, denoted by Sing $S$. As for usual manifolds, the branched manifolds have a well-defined tangent space. For each point $x \in D_{i j}$, the tangent space $T_{x}\left(D_{i j}\right)$ is identified with $T_{\psi_{i}(x)}\left(\mathbb{D}^{p}\right)$ by the isomorphism $\left(\left.\psi_{i}\right|_{D_{i j}}\right)_{* x}$. The tangent bundle $T(S)$ is the quotient of the disjoint union $\bigsqcup T\left(D_{i j}\right)$ by the equivalence relation which identifies $v \in T_{x}\left(D_{i j}\right)$ and $w \in T_{y}\left(D_{k l}\right)$ if $x=y$ and $v=\left(\psi_{i} \circ \psi_{k}^{-1}\right)_{*}(w)$.

A continuous map $f: S \rightarrow S^{\prime}$ between two branched manifolds $S$ and $S^{\prime}$ is of class $C^{r}$ if each function $f_{i j}^{k}=\left.\psi_{k} \circ f\right|_{D_{i j} \cap f^{-1}\left(U_{k}^{\prime}\right)} \circ\left(\left.\psi_{i}\right|_{D_{i j}}\right)^{-1}: \mathbb{D}^{p} \rightarrow \mathbb{D}^{p^{\prime}}$ is of class $C^{r}$ and its germ at any point of $\mathbb{D}^{p}$ does not depend on $j$. The last condition implies that $f$ has the same behavior on different branches around a singular point. Obviously $f$ induces a linear map $f_{* x}: T_{x}(S) \rightarrow T_{f(x)}(S)$ for each $x \in S$. The map $f$ is an immersion (resp. submersion) if $f_{* x}$ is a monomorphism (resp. epimorphism) for each $x \in S$. Finally, the map $f$ is cellular if $f(\operatorname{Sing} S) \subset \operatorname{Sing} S^{\prime}$.

3.3. Inverse limits. Finally, we can complete the proof of Theorem 1.1 For this, we proceed in three steps:

i) Construction of a sequence of branched manifolds $S_{n}$. The idea is very simple: we obtain $S_{n}$ by collapsing each element of $\mathcal{B}^{(n)}$ to a plaque. Indeed, for all $n \geq 1$, we denote by $S_{n}=M / \sim_{n}$ the quotient of $M$ by the equivalence relation $\sim_{n}$ which identifies two points of $M$ if and only if they belong to the same flow box and the same transversal. We may also define $S_{n}$ as the quotient of the manifold $\mathcal{M}$ by the previously defined relation $\sim_{n}$. Therefore $S_{n}$ is a $p$-dimensional branched manifold of class $C^{1}$. It is clear that the canonical projection $\pi_{n}: \mathcal{M} \rightarrow S_{n}$ is a covering map between $p$-dimensional branched manifolds of class $C^{1}$.

ii) Construction of the inverse limit $S_{\infty}$. Since each plaque of $\mathcal{B}^{(n+1)}$ is union of plaques of $\mathcal{B}^{(n)}$, the covering map $\pi_{n}: \mathcal{M} \rightarrow S_{n}$ induces a covering map $f_{n+1}$ : $S_{n+1} \rightarrow S_{n}$. Obviously, this is a cellular immersion. The inverse limit

$$
S_{\infty}=\lim _{\longleftarrow}\left(S_{n}, f_{n}\right)=\left\{\left(\bar{x}_{n}\right) \in \prod_{n \geq 1} S_{n} \mid f_{n+1}\left(\bar{x}_{n+1}\right)=\bar{x}_{n}, \forall n \geq 1\right\} \subset \prod_{n \geq 1} S_{n}
$$

is a Hausdorff compact space.

iii) Construction of a foliated homeomorphism between $M$ and $S_{\infty}$. By the universal property of $S_{\infty}$, there is a map $\pi_{\infty}: M \rightarrow S_{\infty}$ which makes the following 
diagram commutative:

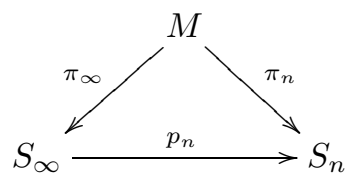

where $p_{n}: S_{\infty} \rightarrow S_{n}$ is the canonical projection. Its image is dense since the maps $\pi_{n}$ are surjective. By compactness of $M, \pi_{\infty}$ is also surjective. Then, to show that $\pi_{\infty}$ is a homeomorphism, it is enough to see that $\pi_{\infty}$ is injective. Remember that the intersection of axes $\mathcal{C}^{(n)}$ is reduced to a point. Therefore, if $x, y \in M$ are different, there is $n \geq 1$ such that $\pi_{n}(x) \neq \pi_{n}(y)$ and then $\pi_{\infty}(x) \neq \pi_{\infty}(y)$.

Finally, for each $x \in \mathcal{M}$ and each integer $n \geq 1$, we will identify

$$
C_{n}^{x}=\left\{\left(\bar{x}_{n}\right) \in S_{\infty} \mid f_{n, m}\left(\bar{x}_{m}\right)=\bar{x}_{n}=\pi_{n}(x), \forall m \geq n\right\}=p_{n}^{-1}\left(\bar{x}_{n}\right)
$$

with the Cantor set $C$, where $f_{n, m}=f_{n} \circ \ldots \circ f_{m}$. If $x$ belongs to the interior $V$ of a plaque $D$ of $\mathcal{B}^{n}$ for some $n \geq 1$ (and thus $\bar{x}_{m} \notin \operatorname{Sing} S_{m}$ for all $m \geq n$ ), then the identification between $C_{n}^{x}$ and $C$ extends to $V$. In other words, for each $y \in V$ there is a unique sequence $\left(\bar{y}_{n}\right) \in C_{n}^{y}$ such that $\bar{x}_{m}$ and $\bar{y}_{m}$ belong to the interior of the same plaque of $S_{m}$ for all $m \geq n$. On the other hand, if $x$ belongs to the boundary of a plaque of $\mathcal{B}^{(n)}$ (and thus $\bar{x}_{n} \in \operatorname{Sing} S_{n}$ ) for all $n$, then the identification between $C_{n}^{x}$ and $C$ also extends to a neighborhood $V$ of $x$ (which meets a finite number of plaques of $\mathcal{B}$ ). This allows us to define a continuous map $q: \mathcal{M} \times C \rightarrow S_{\infty}$. The map $\pi_{\infty}: M \rightarrow S_{\infty}$ described in the diagram (3.4) is then induced by $q$. Since $q$ homeomorphically sends each open set $V \times C$ onto an open subset $U=\left\{\left(\bar{y}_{n}\right) \in S_{\infty} \mid f_{n, m}\left(\bar{y}_{m}\right) \in \pi_{n}(V), \forall m \geq n\right\}$ of $S_{\infty}$, the horizontal lamination of $\mathcal{M} \times C$ induces a lamination of $S_{\infty}$. Now it is clear that $\pi_{\infty}$ is a foliated map preserving the laminations on $M$ and $S_{\infty}$.

Remark 3.5. As mentioned in the introduction, Theorem 1.1 applies to laminations which are not given by free actions of Lie groups. For example, for any finitely generated group, the continuous hull of any aperiodic and repetitive subtree of its Cayley graph [1, 10, 15 is an inverse limit of graphs. In fact, if the group is abelian, the equivalence relation induced on any total transversal is affable, i.e. approximatively finite up to orbital equivalence [2, 15].

\section{Transverse inVARIANT MEASURES}

In the orientable case, we can use the inverse limit structure to describe the set of transverse invariant measures $\mathcal{M}(\mathcal{F})$ extending Theorem 5.1 of $[4]$.

\subsection{Description of the transverse invariant measures.}

Proof of Theorem 1.3. For each $n \geq 1$, the branched manifold $S_{n}$ has a cellular structure induced by the box decomposition $\mathcal{B}^{(n)}$. Although it is not always true, for the sake of simplicity we may suppose that $\mathcal{B}^{(n)}$ is simplicial; that is, any box $B \in \mathcal{B}^{(n)}$ is homeomorphic to a product of a $p$-simplex $\Delta$ of $S_{n}$ and a clopen subset $C$ of the axis $\mathcal{C}^{(n)}$ of $\mathcal{B}^{(n)}$. Let $\mu \in \mathcal{M}(\mathcal{F})$ be a transverse invariant measure defined over any total transversal $\mathcal{C}$. Now we can assign to each $p$-simplex $\Delta$ the measure $\mu(C)$ of the corresponding transversal $C$. For this purpose, we can suppose that 
$C \subset \mathcal{C}$ by sliding $C$ into $\mathcal{C}$. Indeed, the pseudogroup equivalence between the holonomy pseudogroups of $\mathcal{F}$ reduced to $\mathcal{C}^{(n)}$ and $\mathcal{C}$ (see [12]) allows us to replace $C$ with a clopen subset of $\mathcal{C}$ (or equivalently $\mu$ with a measure over $\mathcal{C}^{(n)}$ ). Thus, we obtain a simplicial chain $z_{n} \in C_{p}^{+}\left(S_{n}, \mathbb{R}\right)$. Since $\mu$ is invariant, $z_{n}$ is a cycle. It is enough to observe that if $C$ is a transversal contained in the vertical boundary of a box $B$ and if $C$ decomposes in a finite number of transversals $C_{1}, \ldots, C_{m}$ contained in the vertical boundaries of boxes $B_{1}, \ldots, B_{m} \in \mathcal{B}^{(n)}$, then $\mu(C)=\sum_{i=1}^{m} \mu\left(C_{i}\right)$.

Bearing in mind the properties of the inflation process, we have just defined a linear map $\zeta: \mathcal{M}(\mathcal{F}) \rightarrow \lim H_{p}^{+}\left(S_{n}, \mathbb{R}\right)$ which sends each transverse invariant measure $\mu$ in a sequence $\left(z_{n}\right)$ such that $\left(f_{n, n+1}\right)_{*} z_{n+1}=z_{n}$. Let us show that it is an isomorphism. First of all, again using the fact that the holonomy pseudogroups of $\mathcal{F}$ reduced to $\mathcal{C}^{(n)}$ and $\mathcal{C}$ are equivalent, the decomposition of $\mathcal{C}^{(n)}$ as the disjoint union of the local transversals of the flow boxes $B \in \mathcal{B}^{(n)}$ induces a natural partition $\mathcal{P}^{(n)}$ of $\mathcal{C}$ into clopen subsets. By construction, each partition $\mathcal{P}^{(n)}$ is finer than the previous one; i.e. each element of $\mathcal{P}^{(n)}$ is contained in an element of $\mathcal{P}^{(n-1)}$, and its mesh $\sup \left\{\delta(C) \mid C \in \mathcal{P}^{(n)}\right\}$ converges to 0 . Thus, given a sequence $\left(z_{n}\right) \in$ $\lim _{p} H_{p}^{+}\left(S_{n}, \mathbb{R}\right)$, the coefficients of each chain $z_{n}$ can be interpreted as measures of the elements of $\mathcal{P}^{(n)}$. Then they define a positive Borel measure $\mu$ over $\mathcal{C}$. Any holonomy map can be reduced (by partitioning the domain and the range into clopen subsets small enough to be slid into $\mathcal{C}^{(n)}$ ) to a local homeomorphism between two local transversals in a box of $B^{(n)}$ for a large enough $n>1$. Therefore $\mu$ is holonomy invariant. We have constructed a linear map from $H_{p}^{+}\left(S_{n}, \mathbb{R}\right)$ to $\mathcal{M}(\mathcal{F})$. Finally, it is clear that this map is precisely the inverse map of $\zeta$.

4.2. Ruelle-Sullivan isomorphism. In the situation described above, we can

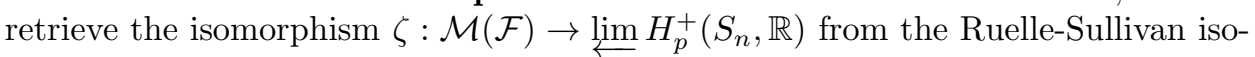
morphism $R S: \mathcal{M}(\mathcal{F}) \rightarrow H_{p}^{+}(\mathcal{F})$ representing every transverse invariant measure as a foliated cycle [17. We first recall that:

i) a foliated $p$-form $\omega \in \Omega^{p}(\mathcal{F})$ is a family of differentiable $p$-forms over the plaques of a foliated atlas continuously depending on the transverse parameter and which agree on the intersection of each pair of flow boxes;

ii) a foliated p-cycle $C \in H_{p}^{+}(\mathcal{F})$ is a continuous linear functional $C: \Omega^{p}(\mathcal{F}) \rightarrow \mathbb{R}$ which is strictly positive on strictly positive forms and null on exact forms (with respect to the leafwise exterior differential $d_{\mathcal{F}}: \Omega^{p-1}(\mathcal{F}) \rightarrow \Omega^{p}(\mathcal{F})$ ).

In other words, $H_{p}^{+}(\mathcal{F})$ is the positive cone of the $p$-th homology group of the foliated currents complex $\left(C_{*}(\mathcal{F}), \partial\right)$, which is the topological dual of the foliated forms complex $\left(\Omega^{*}(\mathcal{F}), d_{\mathcal{F}}\right)$. On the other hand, the classical duality between differential forms and currents [21] makes sense for any branched manifold $S_{n}$. In degree $p$, the epimorphism of complexes $\pi_{*}: C_{*}(\mathcal{F}) \rightarrow \lim C_{*}\left(S_{n}\right)$ (defined by the epimorphisms $\left.\left(\pi_{n}\right)_{*}: C_{*}(\mathcal{F}) \rightarrow C_{*}\left(S_{n}\right)\right)$ induces an isomorphism in homology. According to a classic result of [1], this homology is given by

$$
0 \rightarrow \lim ^{1} H_{r+1}\left(S_{n}, \mathbb{R}\right) \rightarrow H_{r}\left(\varliminf_{\longleftarrow} C_{*}\left(S_{n}\right)\right) \rightarrow \underset{\lim }{\longleftarrow} H_{r}\left(S_{n}, \mathbb{R}\right) \rightarrow 0,
$$

where $\lim ^{1}$ denotes the first derived functor of the inverse limit functor. This provides an isomorphism $H_{p}\left(\lim _{\longleftarrow} C_{*}\left(S_{n}\right)\right) \cong \lim _{\longleftarrow} H_{p}\left(S_{n}, \mathbb{R}\right)$ in the top dimension. 


\section{ACKNOWLEDGMENT}

The authors are grateful for the helpful comments and suggestions made by the referee and especially for pointing out reference [25].

\section{REFERENCES}

[1] F. Alcalde Cuesta, Á. Lozano Rojo, M. Macho Stadler. Dynamique transverse de la lamination de Ghys-Kenyon. Astérisque, 323 (2009), 1-16. MR2647961

[2] F. Alcalde Cuesta, P. González Sequeiros, Á. Lozano Rojo. Affability of Euclidean tilings. C. R. Acad. Sci. Paris, Ser. I, 347 (2009), 947-952. MR2542900

[3] J. Bellissard, R. Benedetti, J.-M. Gambaudo. Spaces of tilings, finite telescopic approximations and gap-labelling. Comm. Math. Phys., 261 (2006), 1-41. MR2193205 (2007c:46063)

[4] R. Benedetti, J.-M. Gambaudo. On the dynamics of $\mathbb{G}$-solenoids. Applications to Delone sets. Ergodic Theory Dynam. Systems, 23 (2003), 673-691. MR1992658(2004f:37019)

[5] M. Bermúdez, G. Hector. Laminations hyperfinites et revêtements. Ergodic Theory Dynam. Systems, 26 (2005), 305-339. MR2218763 (2007d:37033)

[6] E. Blanc. Examples of mixed minimal foliated spaces. Unpublished paper, 2002.

[7] S. S. Cairns. On the triangulation of regular loci. Ann. of Math. (2), 35 (1934), 579-587. MR.1503181

[8] S. S. Cairns. A simple triangulation method for smooth manifolds. Bull. Amer. Math. Soc., 67 (1961), 389-390. MR0149491 (26:6978)

[9] A. Candel, L. Conlon. Foliations I. Graduate Studies in Mathematics, 23, American Mathematical Society, Providence, RI, 2000. MR,1732868 (2002f:57058)

[10] É. Ghys. Laminations par surfaces de Riemann. Panor. Synthèses, 8, Soc. Math. France, Paris, 1999, 49-95. MR:1760843 (2001g:37068)

[11] A. Grothendieck. Éléments de géométrie algébrique (rédigés avec la collaboration de Jean Dieudonné): III. Étude cohomologique des faisceaux cohérents, Première partie. Publications Mathématiques de l'I.H.É.S., 11 (1961), 5-167. MR0217085(36:177c)

[12] A. Haefliger. Foliations and compactly generated pseudogroups, in Foliations: geometry and dynamics (Warsaw, 2000), World Scientific, River Edge, NJ, 2002, 275-295. MR 1882774 (2003g:58029)

[13] J. L. Heitsch, C. Lazarov. Homotopy invariance of foliation Betti numbers. Invent. Math., 104 (1991), 321-347. MR 1098613(92b:58219)

[14] M. W. Hirsch. Differential Topology. Springer-Verlag, New York, 1976. MR0448362 (56:6669)

[15] Á. Lozano Rojo. Dinámica transversa de laminaciones definidas por grafos repetitivos. Ph.D. Thesis, UPV/EHU, 2008.

[16] Á. Lozano Rojo. An example of a non-uniquely ergodic lamination. To appear in Ergodic Theory Dynam. Systems. DOI:10.1017/S0143385710000064

[17] C. C. Moore, C. Schochet. Global Analysis on Foliated Spaces. Second Edition. Mathematical Sciences Research Institute Publications, 9. Cambridge University Press, New York, 2006. MR2202625 (2006i:58035)

[18] J. R. Munkres. Elementary Differential Topology. Revised Edition. Annals of Mathematics Studies, 54, Princeton University Press, Princeton, NJ, 1966. MR0198479 (33:6637)

[19] J. R. Munkres. Topology. Second Edition. Prentice Hall, Upper Saddle River, NJ, 2000. MR 0464128 (57:4063)

[20] A. Phillips, D. Sullivan. Geometry of leaves. Topology, 20 (1981), 209-218. MR605659 (82f:57021)

[21] G. De Rham. Variétés différentiables: formes, courants, formes harmoniques. Hermann, Paris, 1955. MR0346830(49:11552)

[22] W. P. Thurston. The theory of foliations of codimension greater than one. Comment. Math. Helvetici, 49 (1974), 214-231. MR0370619(51:6846)

[23] W. P. Thurston. The Geometry and Topology of Three-Manifolds. Electronic version, 2002. http://www.msri.org/publications/books/gt3m/

[24] A. M. Vershik. A Theorem on Periodical Markov Approximation, in Ergodic Theory, Ergodic Theory and Related Topics (Vitte, 1981), Math. Res., 12, Akademie-Verlag, Berlin, 1981, 195-206. MR730788 (85j:28026) 
[25] A. Weil. Sur les théorèmes de de Rham, Comment. Math. Helvetici, 26 (1952), 119-145. MR0050280 (14:307b)

[26] R. F. Williams. Expanding attractors. Publications Mathématiques de l'I.H.É.S., 43 (1974), 169-203. MR0348794 (50:1289)

[27] J. H. C. Whitehead. On $C^{1}$-complexes. Ann. of Math. (2), 41 (1940), 809-824. MR0002545 $(2: 73 \mathrm{~d})$

[28] H. Whitney. Geometric Integration Theory. Princeton University Press, Princeton, NJ, 1957. MR0087148(19:309c)

Department of Geometry and Topology, University of Santiago de Compostela, 15782 Santiago de Compostela, Spain

E-mail address: fernando.alcalde@usc.es

Department of Mathematics, University of the Basque Country, 48940 Leioa, Spain

E-mail address: alvaro.lozano@ehu.es

URL: http://www.ehu.es/alvaro/

Department of Mathematics, University of the Basque Country, 48940 Leioa, Spain

E-mail address: marta.macho@ehu.es

URL: http://www.ehu.es/〜mtwmastm/ 